\title{
Editorial
}

\section{Medically Unexplained Symptoms and Bodily Distress}

\section{Four Challenges to Improve Understanding and Evidence-Based Care}

\author{
Alexandra Martin ${ }^{1}$ and Omer Van den Bergh ${ }^{2}$ \\ ${ }^{1}$ Department of Clinical Psychology and Psychotherapy, School of Human and Social Sciences, University of Wuppertal, Germany \\ ${ }^{2}$ Health Psychology, KU Leuven - University of Leuven, Belgium
}

Various bodily complaints such as pain, palpitations, fatigue, dizziness, bowel dysfunctions, and others are often considered "medically unexplained symptoms" (MUS), if they are poorly or not related to any observable physiological dysfunction. This type of bodily symptoms is highly prevalent in both children and adults, and is of high relevance for health care. Diagnoses used for this clinical problem are, among others, "Somatic Symptom Disorder" (5th edition of the Diagnostic and Statistical Manual of Mental Disorders, DSM-5; American Psychiatric Association, 2013), "Somatoform Disorders" (10th Revision of the International Classification of Diseases ICD-10, World Health Organization, 1993), "Bodily Distress Disorder" (draft for ICD-11), or functional somatic syndromes. To cover all these conditions, we use the term "bodily distress" in this editorial. Current challenges range from a better understanding of the underlying psychological and pathophysiological mechanisms to a better diagnosis and more effective treatment approaches. This topical issue addresses four specific challenges, which we briefly introduce below.

\section{Challenge 1: Detection and Diagnosis}

Early identification of cases with bodily distress at risk of a chronic course is an important challenge in primary care. Unfortunately, persons at risk are typically underdiagnosed. The group of olde Hartman (olde Hartman et al., 2020) investigated why severe persistent fatigue is underdiagnosed in primary care using a Dutch database with 917 female primary care patients. Interestingly, women with severe persistent fatigue visited their general practitioner (GP) more often than other women, but more than half of these women did not visit their GP with fatigue as the reason and only a minority of them mentioned psychological or social elements with relevance to their condition across four years. This study suggests factors on both the sides of the GP and of the patient that contribute to underdiagnosis of severe persistent fatigue.

\section{Challenge 2: Etiologically Relevant Mechanisms}

Different explanatory approaches have been proposed to account for the development and/or persistence of bodily distress (for recent overviews see, Henningsen, Gündel, et al., 2018; Henningsen, Zipfel, Sattel, \& Creed, 2018; Van den Bergh et al., 2017). One critical question with both theoretical and clinical implications is whether persons with MUS are better or poorer perceivers of their bodily state. Indeed, being interoceptively hypervigilant and tending to catastrophize about bodily sensations could act both ways: It might promote better interoception, but it might also result in misperceiving internal sensations (more false positives). The study of Witthöft, Bräscher, and Köteles (2020) adds an element to a body of evidence that suggests the latter interpretation. Stronger sensory symptom perceptions in the cardiorespiratory system were associated with lower interoceptive accuracy, but only in a heterogeneous group including patients with health anxiety or somatoform disorders. Another study (Pollklas et al., 2020) investigated the impact of searching the internet for health-related information on health concerns ("Cyberchondria"). It turned out in a healthy student group that only 5 min of search for a symptom of personal concern already increased health concerns. Interestingly, this effect was moderated by negative affectivity, but not by illness anxiety. This finding adds to 
a growing body of evidence that searching the internet acts like a two-edged sword: it might have beneficial effects for the majority of people, providing relevant information and inducing sufficient health concern to motivate people to visit their primary care physician, but it might also induce disabling health concerns, excessive worrying and health anxiety. The latter is emphasized in the paper of Rask et al. (2020): excessive Internet browsing can be considered a type of maladaptive reassurance seeking in health anxious people, contributing to the exacerbation or maintenance of health anxiety and bodily distress. The authors point to novel findings regarding memory and mental imagery, and discuss the role of transdiagnostic characteristics in the etiology and maintenance of health anxiety and bodily distress.

\section{Challenge 3: Clinical Management and Effective Treatment}

Considerable evidence demonstrates the positive effects of psychological treatment of bodily distress, specifically for cognitive-behavioral treatment approaches (for an overview, see, e.g., Henningsen, Zipfel, et al., 2018; Martin et al., 2013). Two review papers point to neglected fields. Maass et al. (2020) investigated whether treating health anxiety also impacts symptom reporting. Their metaanalysis showed psychological interventions to be effective in reducing health anxiety with large effect sizes posttreatment and moderate effect sizes at follow-up. Interestingly, psychological interventions showed also moderate effects in reducing somatic symptoms posttreatment. However, the small long-term effects point to a need to further improve the treatment concepts for severe health anxiety. Because of the close relationship between health anxiety and somatic symptoms, future intervention trials should not only focus on the reduction of health anxiety, but also on a better coping with somatic symptoms. Kangas, Kallesøe, and Rask (2020) report on a literature review of conceptual, assessment and treatment issues for children and adolescents suffering from persistent bodily distress. The advantages and experiences of a comprehensive diagnostic assessment prior to treatment are outlined, as well as the prominent role of parental factors. The authors recommend to integrate parents more actively in the treatment of the child's bodily distress, and point to the necessity of developing clinical as well as research guidelines for this specific area.

\section{Challenge 4: Implementation of Treatment}

Obviously, it is not sufficient to know etiological mechanisms and have effective treatment protocols. It is also of paramount importance to reach out to bring convenient treatment facilities to the patient. A stepped, collaborative, coordinated health care "Network for Somatoform and Functional Disorders" (Sofu-Net) of primary care physicians (PCPs), psychotherapists, and inpatient mental health clinics has been designed to improve early detection and treatment for patients with bodily distress (Shedden-Mora et al. 2016). The present evaluation (Shedden-Mora et al., 2020) focuses on the appreciation of the network by the participating partners. Apparently, the interdisciplinary collaboration was highly appreciated by the majority of participants, and especially primary care physicians reported that Sofu-Net facilitated mental health care. However, the results also point to limitations and obstacles regarding the maintenance of such a network. One conclusion here is that health care for patients with somatic symptom disorders can be improved when both the health care system and the individual patient level are adequately addressed. Another study (Schroeder, Achenbach, \& Martin, 2020) investigated in a randomized controlled trial whether a single session intervention would be sufficient to alter the clinical course of patients with non-cardiac chest pain if offered early after exclusion of severe cardiopathology. Whereas actual chest pain reports and pain perceptions improved overall over time, the single session intervention did not have additional benefits beyond standard care. Since health concerns and cardiac attributions following cardiac testing predicted chest pain 6 months later, this study shows how high-risk groups with dysfunctional illness perceptions can be identified early and suggests to target these factors more explicitly in treatment programs.

In sum, the contributions of this topical issue report on recent developments related to concepts, psychological processes, and management of bodily distress. The issue presents work from various research perspectives in psychology and medicine and reflects the necessity of interdisciplinary research to advance our understanding of MUS and bodily distress as a heterogeneous condition. A multitude of etiologic and pathogenic mechanisms and contributing factors play a role, representing a constant challenge to refine models of bodily distress to inform primary and secondary prevention, stepped care intervention, and specialized treatment.

\section{References}

American Psychiatric Association. (2013). Diagnostic and statistical manual of mental disorders (DSM-5) (5th ed.). Arlington, TX: American Psychiatric Publishing.

Henningsen, P., Gündel, H., Kop, W. J., Löwe, B., Martin, A. Rief, W., ... Van den Bergh, O. (2018). Persistent physical symptoms as perceptual dysregulation: A neuropsychobehavioral model and its clinical implications. Psychosomatic Medicine, 80, 422-431. https://doi.org/10.1097/PSY.0000000000000588 
Henningsen, P., Zipfel, S., Sattel, H., \& Creed, F. (2018). Management of functional somatic syndromes and bodily distress. Psychotherapy and Psychosomatics, 87, 12-31. https://doi.org/ 10.1159/000484413

Kangas, M., Kallesøe, K. H., \& Rask, C. U. (2020). Functional somatic syndromes (FSS) in children and adolescents: Conceptual, measurement and treatment issues. Zeitschrift für Psychologie, 228, 81-92. https://doi.org/10.1027/2151-2604/ a000401

Maass, U., Kühne, F., Maas, J., Unverdross, M., \& Weck, F. (2020). Psychological Interventions for health anxiety and somatic symptoms: A systematic review and meta-analysis. Zeitschrift für Psychologie, 228, 68-80. https://doi.org/10.1027/21512604/a000400

Martin, A., Härter, M., Henningsen, P., Hiller, W., Kröner-Herwig, B., \& Rief, W. (2013). Evidenzbasierte Leitlinie zur Psychotherapie somatoformer Störungen und assoziierter Syndrome [Evidenced based guideline for psychotherapy of somatoform disorders and associated syndromes]. Göttingen, Germany: Hogrefe.

olde Hartman, T. C., Scheepers, T., Lucassen, P., \& van Boven, K. (2020). Do women with severe persistent fatigue present with fatigue at the primary care consultation? Zeitschrift für Psychologie, 228, 93-99. https://doi.org/10.1027/2151-2604/ a000402

Pollklas, M., Widemann, L., Lochschmidt, M., Plakhuta, A., \& Gerlach, A. L. (2020). Cyberchondriasis - the effect of searching the internet on health concerns. Zeitschrift für Psychologie, 228, 110-118. https://doi.org/10.1027/2151-2604/a000404

Rask, C. U., Gehrt, T., Rimvall, M. K., \& Frostholm, L. (2020). Health anxiety: Conceptualization and future directions. Zeitschrift für Psychologie, 228, 141-144. https://doi.org/ 10.1027/2151-2604/a000407

Shedden-Mora, M. C., Kubyb, A., Piontekc, K., \& Löwe, B. (2020). Stepped, collaborative, coordinated care for somatoform disorders (Sofu-Net) - a mixed-methods evaluation among health care professionals. Zeitschrift für Psychologie, 228, 119-129. https://doi.org/10.1027/2151-2604/a000405

Shedden-Mora, M. C., Groß, B., Lau, K., Gumz, A., Wegscheider, K., \& Löwe, B. (2016). Collaborative stepped care for somatoform disorders: A pre-post-intervention study in primary care.
Journal of Psychosomatic Research, 80, 23-30. https://doi. org/10.1016/j.jpsychores.2015.11.004

Schroeder, S., Achenbach, S., \& Martin, A. (2020). Efficacy of a psychological single-session treatment in non-cardiac chest pain - are subjective illness representations important predictors? Zeitschrift für Psychologie, 228, 130-140. https://doi.org/ 10.1027/2151-2604/a000406

Van den Bergh, O., Witthöft, M., Petersen, S., \& Brown, R. (2017). Symptoms and the body: Taking the inferential leap. Neuroscience \& Biobehavioral Reviews, 74, 185-203. https://doi.org/ 10.1016/j.neubiorev.2017.01.015

Witthöft, M., Bräscher, A.-K., \& Köteles, F. (2020). Somatic symptom perception and interoception - a latent-variable approach. Zeitschrift für Psychologie, 228, 100-109. https:// doi.org/10.1027/2151-2604/a000403

World Health Organization. (1993). The ICD-10 classification of mental and behavioural disorders: Diagnostic criteria for research. Geneva, Switzerland: WHO.

Published online May 19, 2020

\section{Authorship}

Both editors contributed equally to the preparation of this issue.

\section{Alexandra Martin}

Department of Clinical Psychology and Psychotherapy

School of Human and Social Sciences

University of Wuppertal

Gaußstrasse 20

42119 Wuppertal

Germany

martin@uni-wuppertal.de

\section{Omer Van den Bergh}

Health Psychology

University of Leuven

Tiensestraat 102

3000 Leuven

Belgium

omer.vandenbergh@kuleuven.be 